\title{
Wnt/ $\beta$-catenin signaling mediates the abnormal osteogenic and adipogenic capabilities of bone marrow mesenchymal stem cells from chronic graft-versus-host disease patients
}

\author{
Han-zhou Qi ${ }^{1}$, Yi-ling Ye ${ }^{1}$, Yuan Suo ${ }^{1}$, Hong Qu' ${ }^{1}$ Hai-yan Zhang ${ }^{1}$, Kai-bo Yang ${ }^{1}$, Zhi-ping Fan ${ }^{1}$, Fen Huang ${ }^{1}$, Li Xuan ${ }^{1}$,
} Yan-qiu Chen ${ }^{1}$, Hua Jin (10 ${ }^{1}$ and Qi-fa Liu (1)

\begin{abstract}
Chronic graft-versus-host disease (cGVHD) is the main cause of non-relapse mortality after allogeneic hematopoietic stem cell transplantation (allo-HSCT). Mesenchymal stem cells (MSCs) in bone marrow (BM) remain unclear in the pathophysiology of CGVHD. In this study, we analyzed BM-MSCs from 66 patients after allo-HSCT, including 33 with active CGVHD and 33 without CGVHD. BM-MSCs showed similar morphology, frequency, phenotype, and proliferation in patients with or without CGVHD. MSCs from the active CGVHD group showed a decreased apoptosis rate $(P<0.01)$. Osteogenic capacity was increased while adipogenic capacity was decreased in the active CGVHD MSCs compared with no-cGVHD MSCs. The expressions of osteogenic gene RUNX2 and COL1A1 were higher $(P<0.001)$ while adipogenic gene PPAR- $\gamma$ and FABP4 were lower $(P<0.001)$ in the active $\mathrm{CGVHD}$ MSCs than no-cGVHD MSCs. These changes were associated with the severity of CGVHD $(P<0.0001 ; r=0.534, r=0.476, r=-0.796$, and $r=-0.747$, respectively in RUNX2, COL1A1, PPAR- $\gamma$, and FABP4). The expression of Wnt/ $\beta$-catenin pathway ligand Wnt3a was increased in CGVHD-MSCs. The dysfunction of CGVHD-MSCs could be reversed by Dickkopf related protein 1(DKK1) to inhibit the binding of Wnt3a. In summary, the differentiation of BM-MSCs was abnormal in active CGVHD, and its underlying mechanism is the upregulated of Wnt3a through Wnt/B-catenin signaling pathway of MSCs.
\end{abstract}

\section{Introduction}

Allogeneic hematopoietic stem cell transplantation (allo-HSCT) is widely used in the treatment of hematopoietic and non-hematopoietic diseases. Chronic graftversus-host disease (cGVHD) is a leading cause of nonrelapse mortality after allo-HSCT ${ }^{1-5}$. The clinical symptoms of cGVHD are highly variable, including skin sclerosis, bronchiolitis obliterans, as well as salivary and lacrimal glands ${ }^{6-8}$. cGVHD is an autoimmune-like

Correspondence: Hua Jin (echohua1124@163.com) or

Qi-fa Liu (liuqifa1962@163.com)

'Department of Hematology, Nanfang Hospital, Southern Medical University, Guangzhou, China

These authors contributed equally: Han-zhou Qi, Yi-ling Ye, Yuan Suo, Hong Qu Edited by H.-U. Simon syndrome caused by the interactions of donor $\mathrm{CD} 4^{+} \mathrm{T}$ and $\mathrm{B}$ cells and the production of $\operatorname{IgG}^{9,10}$. However, the pathophysiology of $\mathrm{cGVHD}$ is still not completely understood. Emerging evidence from mice and human studies has demonstrated that abnormalities in the bone marrow niche play an important role in the pathogenesis of cGVHD $^{11,12}$. Mesenchymal stem cells (MSCs) are a form of multipotent adult stem cells that can be isolated from bone marrow $(\mathrm{BM})$ and other tissues ${ }^{13-15}$. MSCs possess the multipotency and immunomodulatory capabilities, including the capacity to differentiate into a variety of cell types such as osteoblasts, chondrocytes, adipocytes, and myoblasts ${ }^{16,17}$, and the capacity to suppress immunological responses such as the suppression of $\mathrm{CD}^{+}{ }^{+} \mathrm{T}$ cells, $\mathrm{CD} 8^{+} \mathrm{T}$ cells and $\mathrm{B}$ cells proliferation and

\section{(c) The Author(s) 2021}

(c) (i) Open Access This article is licensed under a Creative Commons Attribution 4.0 International License, which permits use, sharing, adaptation, distribution and reproduction cc) in any medium or format, as long as you give appropriate credit to the original author(s) and the source, provide a link to the Creative Commons license, and indicate if changes were made. The images or other third party material in this article are included in the article's Creative Commons license, unless indicated otherwise in a credit line to the material. If material is not included in the article's Creative Commons license and your intended use is not permitted by statutory regulation or exceeds the permitted use, you will need to obtain permission directly from the copyright holder. To view a copy of this license, visit http://creativecommons.org/licenses/by/4.0/. 
induction of Tregs $^{18}$. Emerging evidence has demonstrated that MSCs participate in the pathogenesis of autoimmune diseases such as rheumatoid arthritis (RA), systemic lupus erythematosus (SLE), and ankylosing spondylitis (AS) ${ }^{19-21}$. For example, the number of MSCs and the osteogenic capacity increased in the RA mouse model $^{20}$, and MSCs from the patients with SLE had a morphological appearance of senescence and impaired capabilities of differentiation ${ }^{19}$. In regard to the relationship between MSCs and the pathogenesis of GVHD, a few studies reported that the number of MSCs were decreased and differentiation was abnormal in aGVHD patients ${ }^{22,23}$. But it is rarely reported whether MSCs were abnormal in cGVHD patients.

With regard to the underlying mechanism of the abnormality of MSCs in autoimmune diseases, some studies demonstrated that the upregulation of the Wnt/ $\beta$-catenin pathway was associated with the dysfunction of $\mathrm{MSCs}^{24-26}$. In GVHD recipients, the mechanism of MSCs dysfunction is unclear. In the present study, we investigated the dysfunction of MSCs in patients with cGVHD and its underlying mechanism.

\section{Results}

\section{Patient characteristics}

Sixty-six patients were enrolled in this study from January 2017 to February 2019. There were 38 males and 28 females, with a median age of 31 (range: 15-55) years. There were no significant differences in age, gender, primary disease, time post-transplantation, conditioning regimen, transplant type, source of stem cell, GVHD prophylaxis, or grade of acute GVHD between patients with and without cGVHD (Table 1). Among patients with active cGVHD, the median time from HSCT to cGVHD diagnosis was 8 (range: 3.4-20.8) months, and the median time from cGVHD diagnosis to sample collection was 2.2 (range: $0.2-7.7$ ) months. The most frequent organ manifestations of cGVHD were skin (69.7\%), liver (45.5\%), and oral mucosa (42.4\%). Fourteen patients (52.4\%) had more than two organs involved. Clinical manifestations of cGVHD are summarized in Table 2.

\section{Phenotype and frequency of BM-MSCs}

MSCs from patients with active cGVHD, no cGVHD, and healthy donors (HD) showed a similar fusiform morphology (Fig. 1A). To investigate the characteristics of the MSCs, flow cytometry was analyzed the cell surface markers, including CD29, CD44, CD90, CD105, CD19, CD31, CD34, and CD45. The results revealed that active cGVHD-MSCs, no cGVHD-MSCs, and HD-MSCs shared the same expression profile (Fig. 1B). The frequencies of active cGVHD-MSCs and no cGVHD-MSCs were significantly lower than those of HD-MSCs (5, 5, and 9, per $10^{6}$ BMMNCs, $P<0.001$, Fig. $1 C$ ). But there were no
Table 1 Patient characteristics.

\begin{tabular}{|c|c|c|c|}
\hline \multirow[b]{2}{*}{ Characteristic } & \multicolumn{3}{|l|}{ Chronic GVHD } \\
\hline & No $(n=33)$ & Active $(n=33)$ & $P$ \\
\hline Age, median (range), y & $26(15-55)$ & $32(15-55)$ & 0.40 \\
\hline Gender, $n(\%)$ & & & 0.08 \\
\hline Male & $14(42.4)$ & $21(63.6)$ & \\
\hline Female & $19(57.6)$ & $12(36.4)$ & \\
\hline Primary disease, no $(\%)^{\mathrm{a}}$ & & & 0.34 \\
\hline AML & $24(72.7)$ & $19(57.6)$ & \\
\hline ALL & $5(15.2)$ & $11(33.3)$ & \\
\hline Others & $4(12.1)$ & $3(9.1)$ & \\
\hline $\begin{array}{l}\text { Time from HSCT to sample } \\
\text { collection (range), months }\end{array}$ & $12.8(1.5-24.0)$ & $9.5(3.6-21.1)$ & 0.11 \\
\hline Conditioning regimen, no $(\%)^{\mathrm{b}}$ & & & 0.14 \\
\hline Myeloablative & $1648.5)$ & $22(66.7)$ & \\
\hline Intensified & $17(51.5)$ & $11(33.3)$ & \\
\hline HLA type, no(\%) & & & 0.13 \\
\hline MSD & $13(39.4)$ & $17(51.5)$ & \\
\hline MUD & $6(18.2)$ & $1(3.0)$ & \\
\hline$H I D$ & $14(42.4)$ & $15(45.5)$ & \\
\hline Source of stem cell, no (\%) & & & 0.13 \\
\hline PBSC & $20(60.6)$ & $19(57.6)$ & \\
\hline $\mathrm{BM}+\mathrm{PBSC}$ & $13(39.4)$ & $14(42.4)$ & \\
\hline GVHD prophylaxis, no $(\%)^{c}$ & & & 1.00 \\
\hline ATG based & 16 & 16 & \\
\hline Non-ATG based & 17 & 17 & \\
\hline $\begin{array}{l}\text { History of acute GVHD } \\
\text { grade, no (\%) }\end{array}$ & & & 0.80 \\
\hline $0-1$ & $15(45.5)$ & $14(42.4)$ & \\
\hline $2-4$ & $18(54.5)$ & 19 (57.6) & \\
\hline $\begin{array}{l}\text { No. of immunosuppressive } \\
\text { therapies before inclusion (\%) }\end{array}$ & & & $<0.001$ \\
\hline None & $20(60.6)$ & $0(0.0)$ & \\
\hline 1 & 11 (33.3) & $4(12.1)$ & \\
\hline 2 & $2(6.1)$ & $15(45.5)$ & \\
\hline$\geq 3$ & $0(0.0)$ & $14(42.4)$ & \\
\hline
\end{tabular}

AML acute myeloid leukemia, ALL acute lymphocytic leukemia, HSCT hematopoietic stem cell transplantation, HLA human leukocyte antigen, MSD HLAmatched sibling donor, MUD HLA-matched unrelated donor, HID haplo-identical donor, $P B S C$ peripheral blood stem cells, $B M$ bone marrow, GVHD graft-versushost disease, ATG antithymocyte globulin.

${ }^{a}$ MThe other category included mixed lineage acute leukemia, myelodysplastic syndrome, and lymphoma.

${ }^{\mathrm{b}}$ Myeloablative conditioning regimens include TBI (total body irradiation) $+\mathrm{Cy}$ (cyclophosphamide), Bu (busulfan) $+\mathrm{Cy}$, and $\mathrm{Bu}+\mathrm{Flu}$ (fludarabine).Intensified conditioning regiments include $\mathrm{TBI}+\mathrm{Cy}+$ etoposide, and Flu + cytarabine + $\mathrm{TBI}+\mathrm{Cy}$.

'Non-ATG based GVHD prophylaxis include cyclosporine A ( $\mathrm{Cs} A)$,methotrexate (MTX), and mycophenolate mofetil (MMF). ATG based GVHD prophylaxis include CsA + MTX + MMF + ATG.

Immunosuppressive treatments include $\mathrm{CsA}$, tacrolimus (Tac), MMF, and steroids.

differences among the frequencies of different severities in active cGVHD-MSCs and no cGVHD-MSCs (5, 5, and 5, per $10^{6}$ BMMNCs, $P=0.57$, Fig. $1 D$ ).

\section{Proliferation and apoptosis of MSCs}

There was no difference in proliferation among active cGVHD-MSCs, no GVHD-MSCs, and HD-MSCs by detecting the passage time from $\mathrm{P} 0$ to $\mathrm{P} 4(P=0.36,0.42$, 
Table 2 Clinical manifestations of cGVHD.

\begin{tabular}{llll}
\hline Organ & $\begin{array}{l}\text { Mild } \\
\mathbf{N = 8}\end{array}$ & $\begin{array}{l}\text { Moderate } \\
\mathbf{N = 1 1}\end{array}$ & $\begin{array}{l}\text { Severe } \\
\mathbf{N}=\mathbf{1 4}\end{array}$ \\
\hline Skin (\%) & $5(15.2)$ & $5(15.2)$ & $13(39.4)$ \\
Liver (\%) & $3(9.1)$ & $6(18.2)$ & $6(18.2)$ \\
Gastrointestinal (\%) & $0(0.0)$ & $0(0.0)$ & $3(9.1)$ \\
Oral mucosa (\%) & $3(9.1)$ & $4(12.1)$ & $7(21.2)$ \\
Eye (\%) & $2(6.1)$ & $0(0.0)$ & $2(6.1)$ \\
Lung (\%) & $0(0.0)$ & $0(0.0)$ & $3(9.1)$ \\
Joint (\%) & $0(0.0)$ & $1(3.0)$ & $0(0.0)$ \\
Genital Tract (\%) & $0(0.0)$ & $1(3.0)$ & $0(0.0)$ \\
\hline
\end{tabular}

0.60, and 0.37, respectively, Fig. 2A). These similarities were also found between patients with mild cGVHD and patients with moderate/severe cGVHD $(\mathrm{P}=0.36,0.70$, 0.96, and 0.91, respectively, Fig. 2B). These findings were also confirmed by CCK-8 cell growth assays (Supplemental Fig. 1). The percentage of early apoptotic MSCs decreased in patients with active cGVHD compared with those of patients without cGVHD $(11.08 \%$ vs. $14.07 \%$, $P<0.01)$ and those of healthy donors $(11.08 \%$ vs. $15.50 \%$, $P<0.01$, Fig. 2 C). These decreases were associated with disease severity. Moderate/severe cGVHD-MSCs had a significantly decreased early apoptotic rate compared with mild cGVHD-MSCs (median, $7.71 \%$ vs. $13.37 \%, P<$ 0.05, Fig. 2D). Comparisons of late apoptosis showed a similar trend. Patients with active cGVHD had significantly lower percentage of late apoptotic MSCs than those of patients without cGVHD (median, $1.25 \%$ vs. $2.01 \%, P<0.01$ ) and those of healthy donors (median, $1.25 \%$ vs. $2.41 \%, P<0.0001$, Fig. 2 C). Further, moderate/ severe cGVHD-MSCs had a significantly decreased late apoptotic rate compared with no cGVHD-MSCs $(1.09 \%$ vs $2.01 \%, P<0.0001$, Fig. $2 \mathrm{D})$.

\section{Differentiation of MSCs}

To investigate whether the differentiation capacity of MSCs in active cGVHD patients, we induced MSCs into osteogenic and adipogenic lineages in vitro. The calcium nodules increased in patients with active cGVHD compared with those of patients without cGVHD and healthy donors. In contrast, the lipid droplets in the active cGVHD group decreased compared with those of nocGVHD group and HD group (Fig. 3A).

The expression of specific marker genes was further investigated using real-time qPCR. The expressions of the osteogenic markers RUNX2 and COL1A1 were significantly increased in active cGVHD-MSCs compared with no cGVHD-MSCs $(1.31$ vs $0.94, P<0.001$ and 1.66 vs $1.29, P<0.001$, respectively) and HD-MSCs (1.31 vs $0.88, P=0.009$; and 1.66 vs $1.27, P=0.023$, respectively). These increases were more significant in patients with moderate/severe cGVHD than in patients without cGVHD (median, 1.32 vs $0.94, r=0.534, P<$ $0.001 ; 1.83$ vs $1.29, r=0.476, P<0.001$, Fig. $3 \mathrm{~B})$. In contrast, the expressions of adipogenic markers PPAR$\gamma$ and FABP4 were decreased in active cGVHD-MSCs compared with that of no cGVHD-MSCs (3.5 vs 5.21, $P<0.001$ and 1.48 vs $3.71, P<0.001$, respectively) and HD-MSCs (3.5 vs $5.74, P<0.001$; and 1.48 vs $3.28, P<$ 0.001 , respectively). Moreover, these decreases were associated with cGVHD severity. Moderate/severe cGVHDMSCs had lower expression of PPAR- $\gamma$ and FABP4 compared to mild cGVHD-MSCs (2.73 vs 4.03, $P=0.017$; and 1.13 vs $2.95, P<0.001$, respectively) and no cGVHD-MSCs (median, 2.73 vs $5.21, P<0.001$; and 1.13 vs $3.71, P<0.001$, respectively, Fig. $3 \mathrm{C}$ ).

\section{Upregulation of the Wnt/ $\beta$-catenin pathway in active cGVHD-MSCs}

To determine whether the Wnt/ $\beta$-catenin pathway mediates the abnormal differentiation of BM-MSCs, we detected the protein expression of the Wnt/ $\beta$-catenin signaling pathway related proteins Wnt3a, GSK-3 $\beta$, and $\beta$-catenin. Our results showed that the Wnt/ $\beta$-catenin pathway ligand Wnt3a in active cGVHD-MSC was significantly increased compared to no cGVHD-MSCs $(0.95$ vs $0.59, P<0.001)$ and HD-MSCs $(0.95$ vs $0.62, P<0.001)$. The level of Wnt3a in moderate/severe cGVHD-MSCs was significantly higher than that of patients with mild cGVHD (1.01 vs $0.79, P<0.001)$. Correspondingly, the phosphorylated/total GSK-3 $\beta$ was increased in active cGVHD-MSCs compared to no cGVHD-MSCs (0.89 vs $0.48, P<0.001)$ and HD-MSCs $(0.89$ vs $0.43, P<0.001)$. The rate of phosphorylated/total $\beta$-catenin was decreased in active cGVHD-MSC compared to no cGVHD-MSCs (0.33 vs $0.48, P<0.001)$ and HD-MSCs $(0.33$ vs $0.46, P<$ 0.001, Fig. 4A-C).

Since upregulation of $\mathrm{Wnt} / \beta$-catenin signaling was found in active cGVHD-MSCs, we next evaluated the effects of inhibiting the Wnt/ $\beta$-catenin pathway by DKK1 on the differentiation capacities of active cGVHD-MSCs in vitro. Our results showed that DKK1 could inhibit the levels of Wnt3a and phospho-GSK3 $\beta$ expression and elevate the level of phospho- $\beta$-catenin expression (Fig. 5A, B). After treated with DKK1, the calcium nodules were decreased and the lipid droplets were increased in active cGVHD-MSCs (Fig. 5C). The levels of osteogenic specific genes RUNX2 and COL1A1 were decreased after DKK1 addition (1.23 vs $1.35, P<$ 0.01 and 1.39 vs $1.66, P<0.05$, respectively). In contrast, the levels of adipogenic specific genes PPAR $\gamma$ and FABP4 were found increased in the DKK1 treated group 


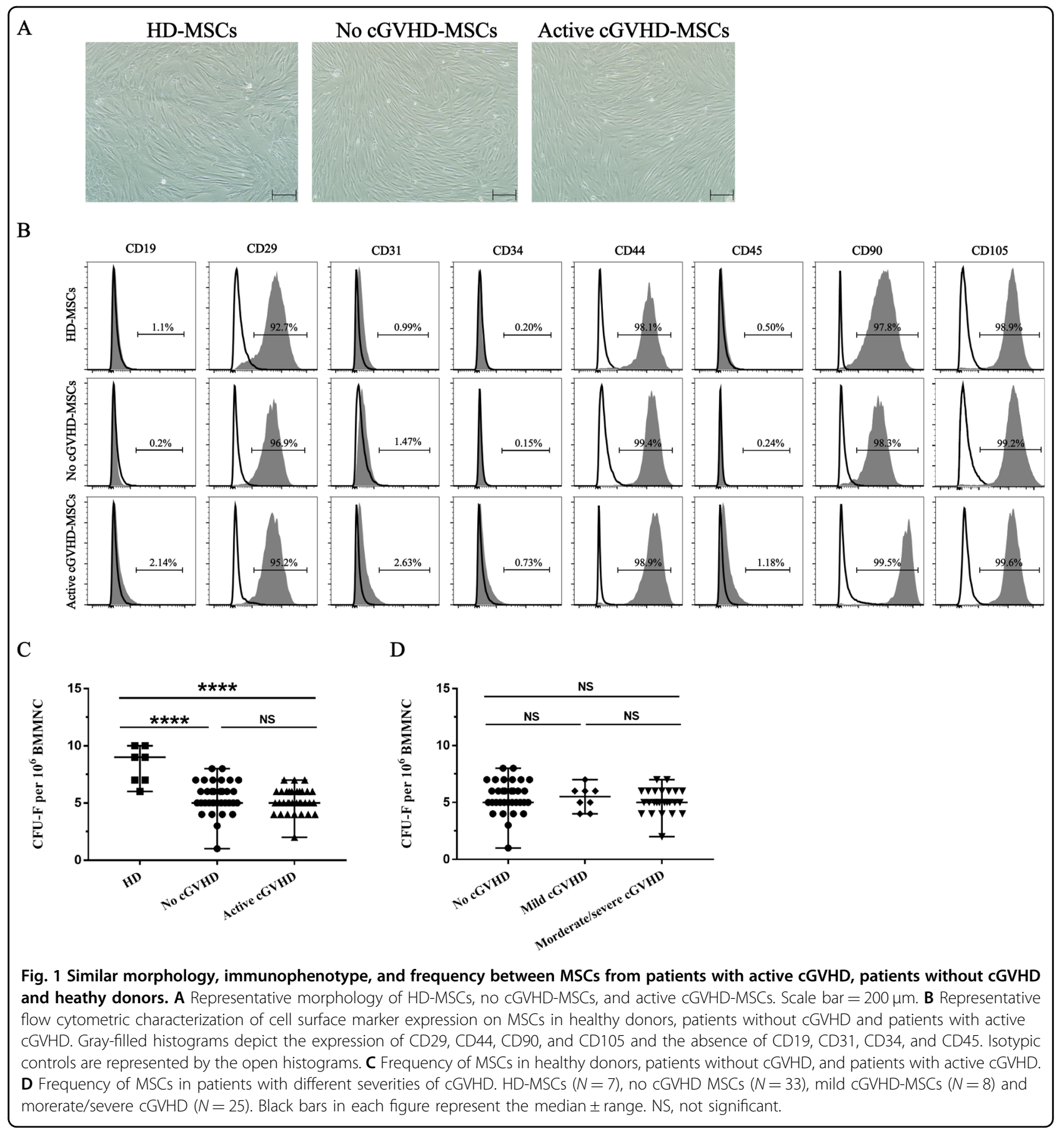

(5.18 vs $4.50, P<0.001$ and 3.32 vs $1.68, P<0.001$, respectively).

Previous studies indicated that apart from Wnt/ $\beta$-catenin pathway, there were several canonical pathways related to the osteogenic and adipogenic differentiation of MSCs, such as Akt, MAPK and Hedgehog pathway ${ }^{27-29}$. To further clarify whether these pathways play a role in the abnormal differentiation of active cGVHD-MSC, we then assayed the expression of proteins regulated by these pathways, Including Akt, p38 MARK and Sonic Hedgehog (SHH). The results showed that there were no significant difference in Akt, p38 MARK and SHH protein expression among BM-MSCs from HD donors, non-cGVHD patients and active cGVHD patients. And they were similar between BM-MSCs in different severities of active cGVHD patients (all $P>0.05$, Supplemental Fig. 3A-C). 


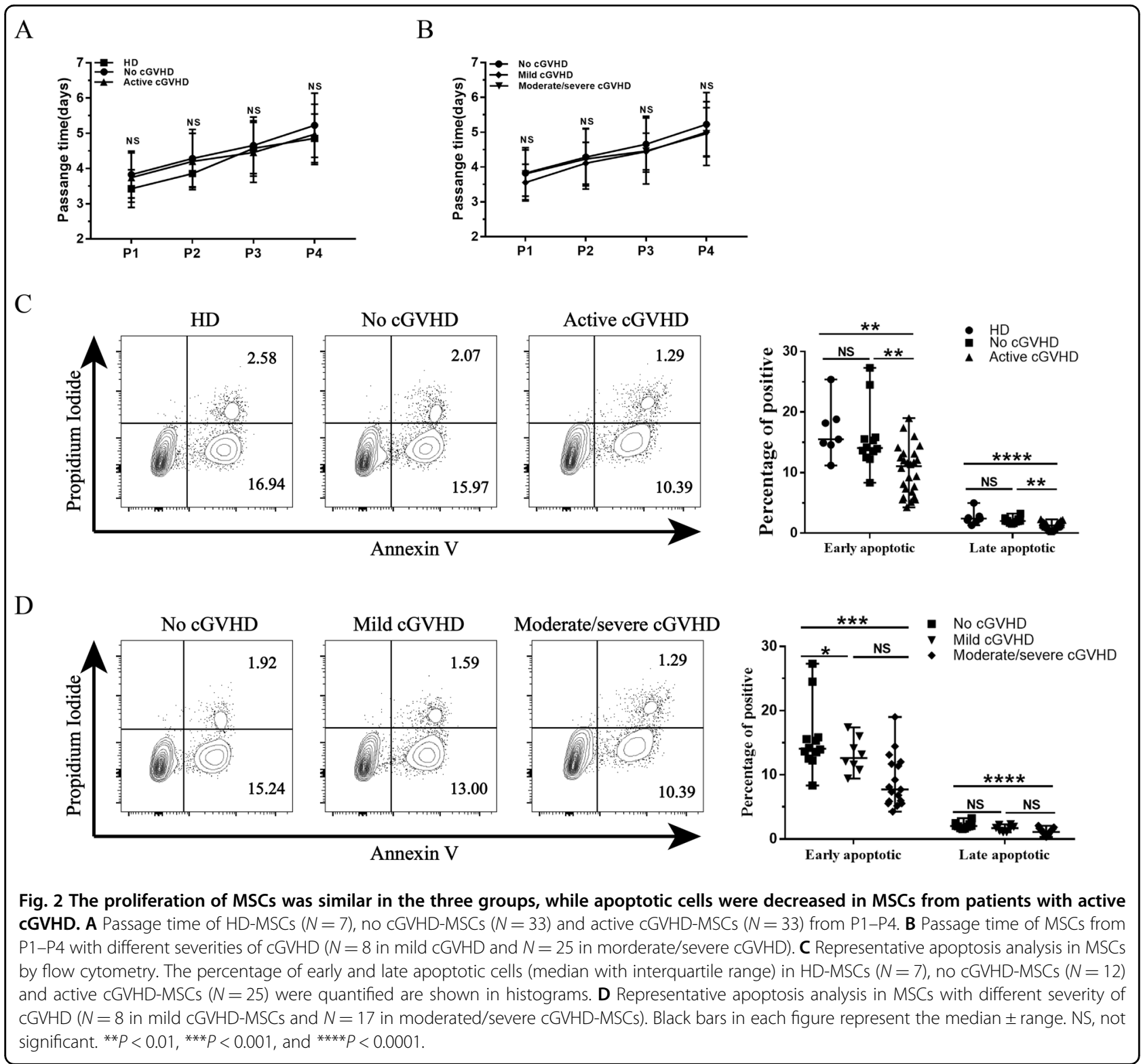

\section{Immunomodulation of active cGVHD-MSCs was comparable to no cGVHD-MSCs and HD-MSCs}

To further investigated the immunomodulatory function of BM-MSCs in active cGVHD patients, we evaluate the cytokine infiltration in the bone marrow serum of active cGVHD patients. The results showed that the infiltration of TNF- $\alpha$ and TGF- $\beta$ was significantly higher in active cGVHD cohort compared to those in no cGVHD cohort (105.4 vs 46.46, pg/ml, $P<0.01$ and 64.68 vs 42.80 , $\mathrm{ng} / \mathrm{ml}, P<0.01$, respectively, Fig. $6 \mathrm{~A})$. The levels of IFN- $\gamma$, IL-17, and IL-6 in active CGVHD cohort showed increasing trends compared to no cGVHD cohort, but did not reach statistical significance (all $P>0.05$, Fig. 6A). These increases were related to the severities of cGVHD, the infiltration of IL- 6 , IL-17, TNF- $\alpha$, IFN- $\gamma$, and TGF- $\beta$ was significantly higher in the bone marrow serum of moderated/severe cGVHD patients compared to no cGVHD patients (IL6: 1.09 vs $0.47, \mathrm{pg} / \mathrm{ml}, P<0.05$; TNF$\alpha: 142.00$ vs $44.47, \mathrm{pg} / \mathrm{ml}, P<0.01$; IFN- $\gamma: 55.36$ vs 17.12 , $\mathrm{pg} / \mathrm{ml}, P<0.001$; IL17:6.19 vs $3.28, \mathrm{pg} / \mathrm{ml}, P<0.01$; TGF$\beta: 72.72$ vs $42.32, \mathrm{ng} / \mathrm{ml}, P<0.001$; Supplemental Fig. $4 \mathrm{~A})$. Previous studies indicated that the immunomodulation of MSCs was affected through cell-cell contact and/or cytokines secretion. Our results showed that whether in cell-cell cocultured or MSCs paracrine cultured (Fig. 6B), the levels of IL-6, IL-10, IL-17, TGF- $\beta 1$, IFN $\gamma$, and TNF $\alpha$ in culture medium were similar among HD-MSCs, non-cGVHD MSCs and active cGVHD MSCs (all $p>0.05$, Fig. 6C). And the capacities of inhibit mixed lymphocyte response (MLR) were similar among 


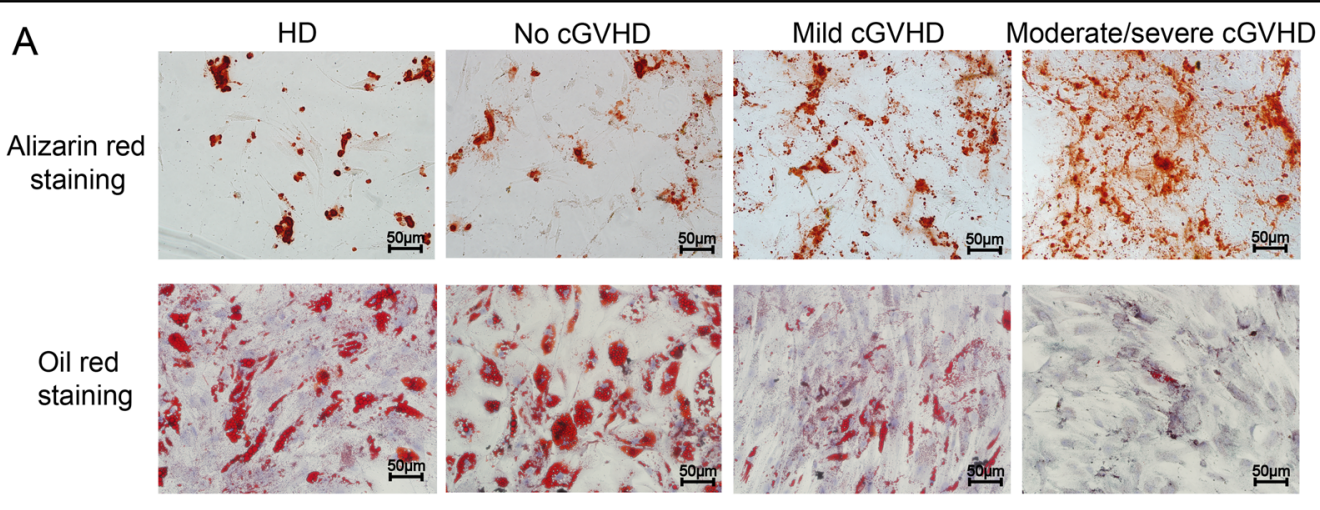

B

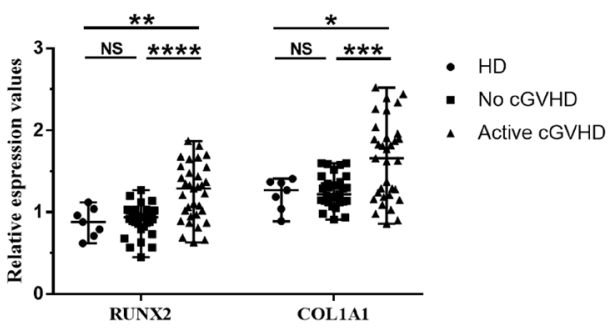

C

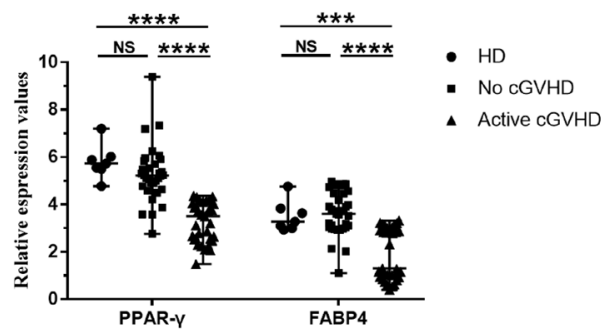

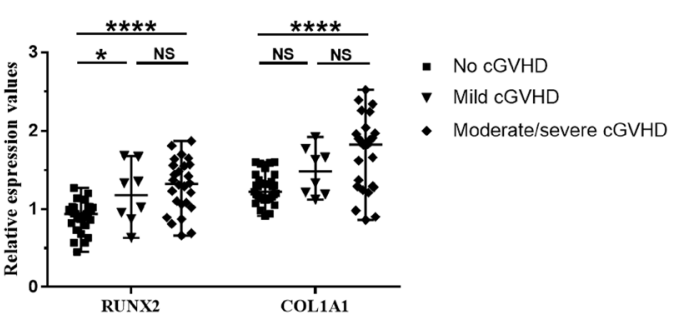

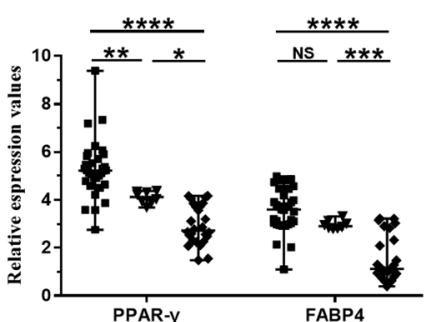

- No cGVHD

- Mild cGVHD

- Moderate/severe cGVHD

Fig. 3 Increased osteogenic differentiation capacity and decreased adipogenic differentiation capacity in MSCs from patients with active cGVHD. A Osteogenic differentiation (above) of HD-MSCs, no cGVHD-MSCs, mild cGVHD-MSCs and moderate/severe cGVHD-MSCS was demonstrated by alizarin red staining, Orange-red represents calcium nodules. Adipogenic differentiation (under) of HD-MSCs, no cGVHD-MSCs, mild cGVHD-MSCs, and moderate/severe CGVHD-MSCs was detected by Oil Red O staining, and red represents lipid droplets. Scar bar $=50 \mu \mathrm{m}$. B Gene expression of osteogenic markers RUNX2 and COL1A1 in HD-MSCs, no CGVHD-MSCs, active CGVHD-MSCs, and different severities of cGVHD-MSCs. C Gene expression of adipogenic markers PPAR- $\gamma$ and FABP4 in HD-MSCs, no CGVHD-MSCs, active CGVHD-MSCs, and different severities of cGVHDMSCs. HD-MSCs $(n=7)$, no cGVHD-MSCs $(n=33)$, mild cGVHD-MSCs $(n=8)$, and moderate/severe cGVHD-MSCs $(n=25)$. Results were normalized to the expression of glyceraldehyde 3-phosphate dehydrogenase (GAPDH). Black bars in each figure represent the median \pm range. NS, not significant. ${ }^{*} P<0.05,{ }^{* *} P<0.01,{ }^{* *} P<0.001$, and ${ }^{* * *} P<0.0001$.

three groups (all $p>0.05$, Fig. 6D, E). Specific to different severities of cGVHD, the level of IL-10 was higher in no cGVHD group compared to morderate/severe cGVHD group when cell-cell coculture with MLR (83.99 vs 63.75, pg/ml, $P<0.05$, Supplemental Fig. 4A). The other cytokines and the inhibition of MLR were similar in different severities of cGVHD-MSCs (all $p>0.05$, Supplemental Fig. 4A-C). It has been documented that NF$\kappa B$ activation mediates the cytokine factors secretion by MSCs. We then assay the activation of NFkB. The results showed that the levels of $\mathrm{p}-\mathrm{NFkB} / \mathrm{NFkB}$ in BM-MSCs showed no difference among three groups $(P>0.05$, Fig. 6F and Supplemental Fig. E).

\section{Discussion}

Our current study demonstrates that the osteogenic and adipogenic capacities of BM-MSCs were impacted in the patients with active cGVHD. Upregulation of the Wnt/ $\beta$-catenin pathway participates in the dysfunction of MSCs in the patients with active cGVHD.

A growing number of studies from animal and human has demonstrated that the dysfunctions of MSCs are associated with autoimmune diseases. Recently, Ding et al. reported that the numbers of MSCs were decreased in the early period after allo-HSCT, then slowly increased over time following allo-HSCT ${ }^{30}$. It has also been reported that the self-renew and differentiation capacities were 

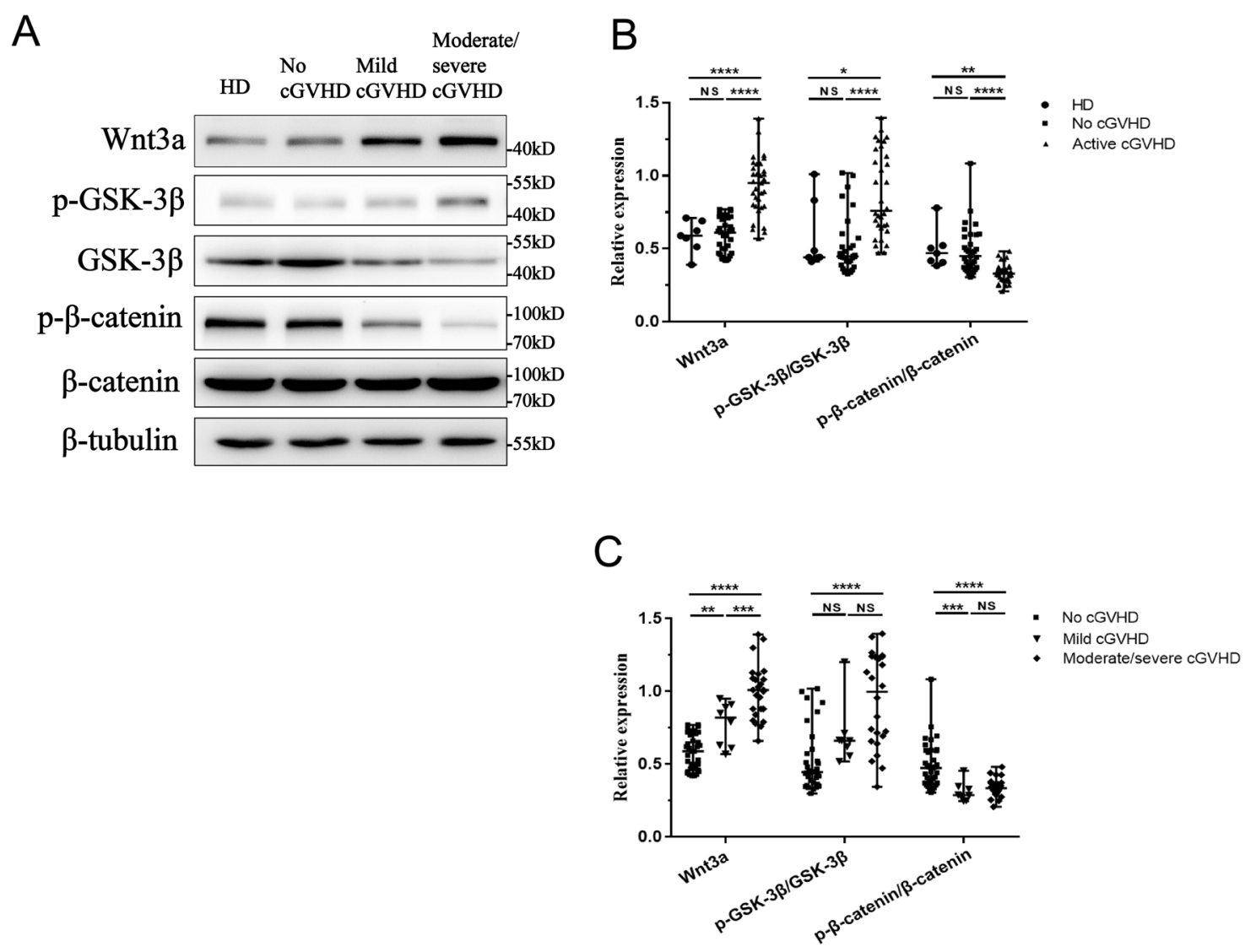

Fig. 4 Upregulation of the Wnt/ $\beta$-catenin pathway in MSCs from patients with active cGVHD. A Representative protein expression of Wnt3a, phosphor-GSK-3 $\beta$, total-GSK-3 $\beta$, phosphor- $\beta$-catenin, total- $\beta$-catenin, and $\beta$-tubulin in MSCs derived from healthy donors, patients without $c$ GVHD, patients with mild-cGVHD and patients with moderate/severe CGVHD. B Relative protein expression of HD-MSCs, no cGVHD-MSCs, and active CGVHD-MSCs. C Relative protein expression of MSCs with different severities of CGVHD.Protein expression was normalized within each sample to the internal reference protein $\beta$-tubulin. HD-MSCs $(n=7)$, no cGVHD-MSCs $(n=33)$, mild cGVHD-MSCs $(n=8)$, and moderate/severe cGVHD-MSCs $(n=$ 25). Black bars in each figure represent the median \pm range. NS, not significant. ${ }^{*} P<0.05,{ }^{* *} P<0.01,{ }^{* *} P<0.001$, and ${ }^{* * *} P<0.0001$.

decreased in the patients with aGVHD compared with the patients without $\mathrm{GVHD}^{23}$. Wang et al. recently reported that the morphology, immunophenotype, self-renewal, and differentiation capacity of BM-MSCs were similar between the patients with cGVHD and those without $\mathrm{cGVHD}^{31}$. In this study, we found the frequencies of MSCs in the patients whether with or without cGHVD after allo-HSCT were decreased compared to heathy donors, while were similar between the patients with active cGVHD and the patients without cGVHD.

Immunomodulation is a key function of MSC through paracrine or/and cell-cell contact in $\mathrm{HSCT}^{32}$. Our study revealed the severity of inflammatory infiltration was significantly enhanced in the bone marrow serum of moderate/severe cGVHD patients compared to that of no cGVHD patients. The NF- $\mathrm{KB}$ signaling pathway plays a critical role in the expression of cytokines and regulates immune responses. It has been reported that NF- $\mathrm{kB}$ activation mediates cytokine factor secretion by $\mathrm{MSCs}^{33,34}$.
In this study, we found that the immune regulation function of BM-MSCs did not affect through the activation of NF- $\mathrm{KB}$ by the inflammatory infiltration in bone marrow serum. The paracrine and immunosuppressive abilities showed no difference between active cGVHDMSCs, no cGVHD-MSCs, and HD-MSCs, no matter MSCs were cultured alone or cocultured with MLR.

With respect to the differentiation functions of MSCs, we found the adipogenic capacity of MSCs was markedly decreased in the patients with active cGVHD, while osteogenic differentiation capacity was increased in the patients with active CGVHD compared with those without cGVHD. Moreover, we further demonstrated that these dysfunctions of MSCs were correlated with the clinical grade of cGVHD. Our findings were inconsistent with Wang's study ${ }^{31}$, in which the reason might be that: (1) The time after transplantation in our patients was shorter than Wang's study (median, 9.5months vs20 months). (2) In our study, the history of II-IV aGVHD was similar 


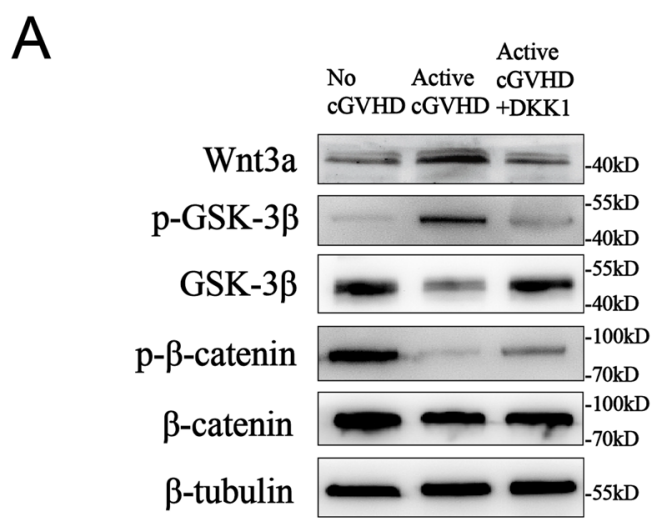

B

C

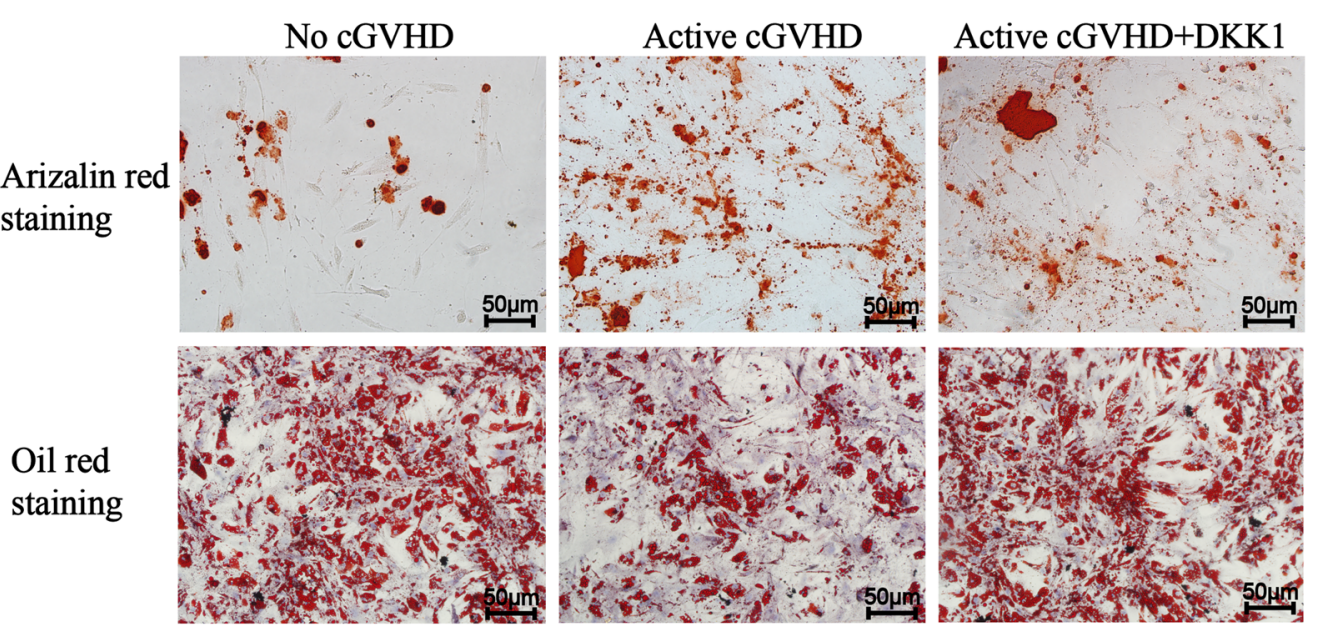

$\mathrm{D}$
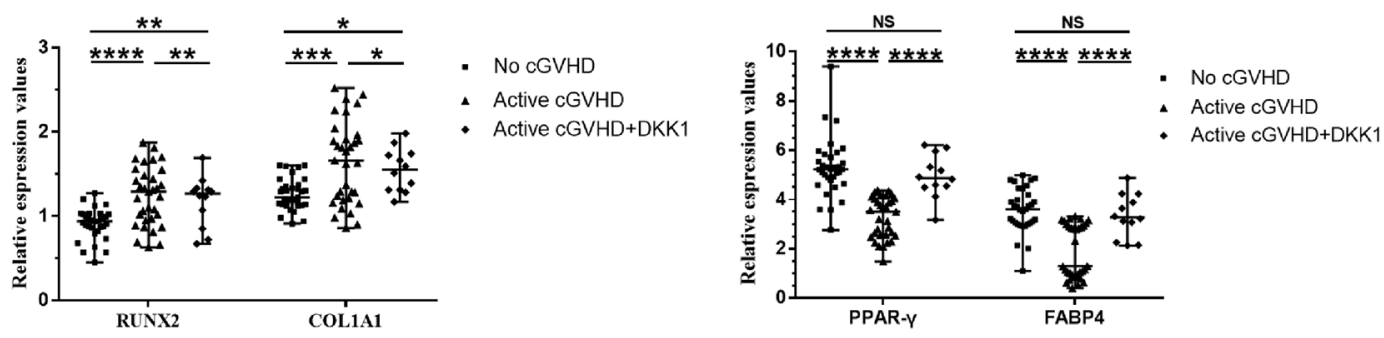

Fig. 5 DKK1 decreased the expression of Wnt/ $\boldsymbol{\beta}$-catenin signaling in active cGVHD-MSCs. A Representative protein expression of Wnt3a, phosphor-GSK-3 $\beta$, total-GSK-3 $\beta$, phosphor- $\beta$-catenin, total- $\beta$-catenin, and $\beta$-tubulin in active cGVHD-MSCs treated or untreated with DKK1 $(n=12)$ and no cGVHD-MSCs $(n=12)$. B Relative protein expression of active cGVHD-MSCs treated or untreated with DKK1 $(n=12)$ and no cGVHD-MSCs $(n=12)$. C Relative gene expression of osteogenic/adipogenic marker gene. D Alizarin red staining, Orange-red represents calcium nodules. Oil Red O staining, red represents lipid droplets. No cGVHD-MSCs $(n=33)$, active cGVHD $(n=33)$ and DKK1 + cGVHD $(n=12)$. Black bars in each figure represent the median \pm range. NS, not significant. ${ }^{*} P<0.05$, ${ }^{* *} P<0.01$, ${ }^{* *} P<0.001$, and ${ }^{* * *} P<0.0001$.

between active cGVHD patients and no cGVHD patients (54.5\% vs $57.6 \%, P=0.80$ ), However, there is a higher incidence of II-IV aGVHD history in cGVHD cohort compared to no cGVHD cohort $(44.0 \%$ vs $12.5 \%, P=$ 0.034) in Wang's study.

Wnt/ $\beta$-catenin signaling is an evolutionarily conserved intracellular signaling and plays an important role in the osteogenic and adipogenic differentiation of $\mathrm{MSCs}^{35,36}$. Activation of $\mathrm{Wnt} / \beta$-catenin signaling is related to the decrease of osteogenic differentiation and the increase of adipogenic differentiation ${ }^{37,38}$. Briefly, the increased of Wnt3a upregulated the phosphorylation of GSK3 $\beta$, and p-GSK3 $\beta$ subsequently resulted in nuclear $\beta$-catenin accumulation, induced the promotion of osteogenic and 

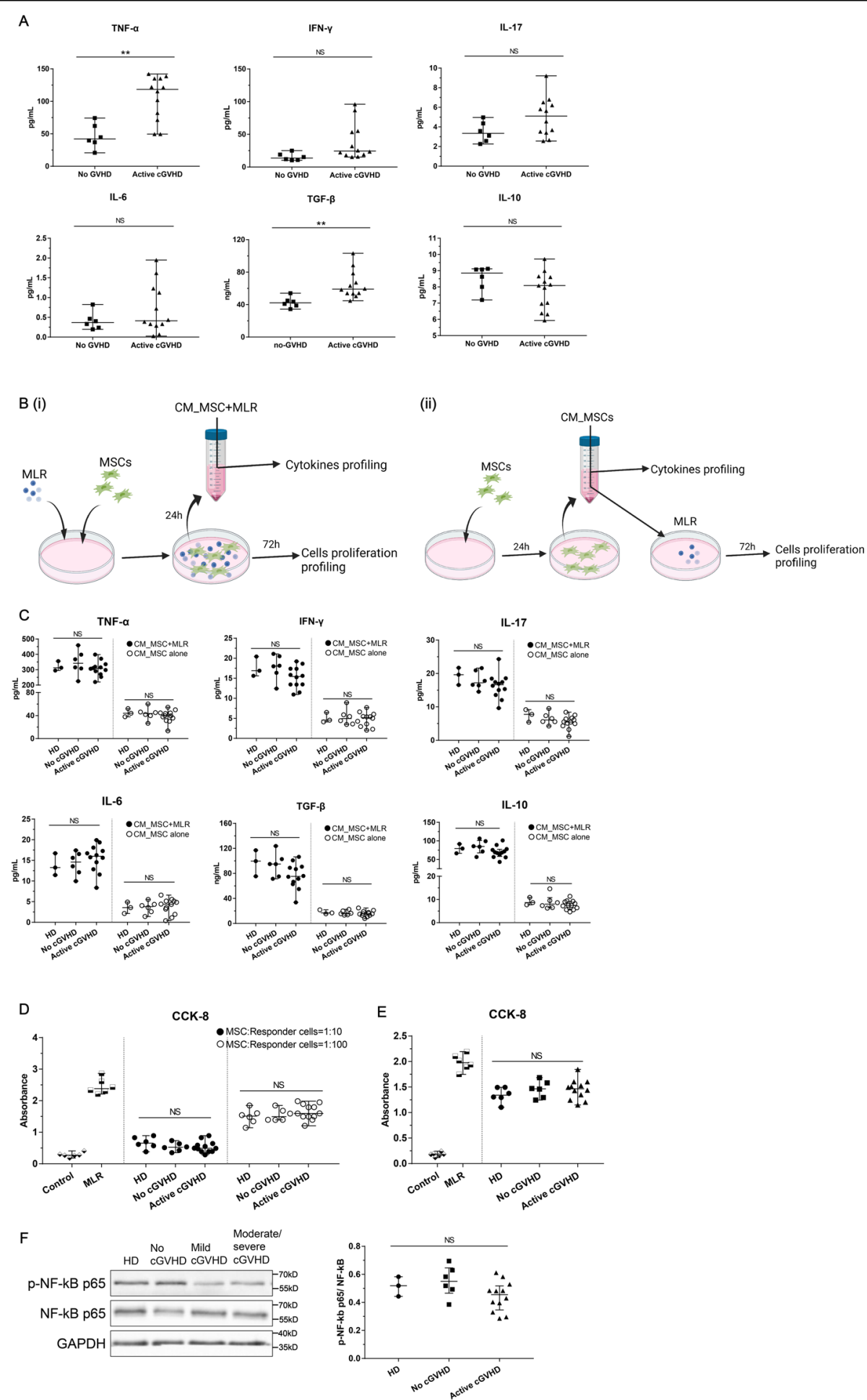

Fig. 6 Immunomodulation of MSCs. A The levels of IL-6, IL-10, IL-17, TNFa, IFN-r, and TGF-b in bone marrow serum from no cGVHD and active cGVHD patients. B Schematic diagram showing (i) the MSC co-cultrued with MLR conditioned medium collection process and (ii) CM_MSC cultrued with MLR conditioned medium collection process. C The levels of IL-6, IL-10, IL-17, TNFa, IFN-r, and TGF-b in the conditioned medium of cell-cell cocultured and MSCs cultured alone. D The immunosuppressive function of different concentrations of cocultured MSCs from HD, no cGVHD, and active CGVHD patients. $\mathbf{E}$ The paracrine immunosuppressive function of MSCs conditioned medium with MLR. $\mathbf{F}$ Representative protein expression of NF-kB, p-NF-kB, and GAPDH in MSCs derived from healthy donors, patients without cGVHD and patients with active cGVHD. HD-MSCs $(n=3)$, no cGVHD-MSCs $(n=6)$, and active CGVHD-MSCS $(n=12)$. Black bars in each figure represent the median \pm range. NS, not significant. ${ }^{*} P<0.05$, ${ }^{* *} P<$ $0.01,{ }^{* *} P<0.001$, and ${ }^{* * *} P<0.0001$. 
inhibition of adipogenic. The role of $\mathrm{Wnt} / \beta$-catenin signaling in the pathogenesis of autoimmune diseases is gaining more and more attention. Nakamura et al. reported that the Wnt/ $\beta$-catenin ligands were significantly upregulated in RA synovium ${ }^{39}$. Briolay et al. reported that in patients with ankylosing spondylitis, $\mathrm{Wnt} / \beta$-catenin signaling activation might be involved in the effects of inflammation on bone formation by promoted MSCs osteogenic differentiation $^{25}$. Wang et al. revealed an elevated Wnt/ $\beta$-catenin signaling activity in human renal tissues of patients with lupus nephritis(LN), suggesting that a dysregulated $\mathrm{Wnt} / \beta$-catenin signaling might play a role in the renal fibrosis ${ }^{40}$. Recently, Chen et al. reported that Wnt/ $\beta$-catenin signaling pathway was implicated in the activation of fibrogenic program in fibroblasts in cGVHD mice model $^{41}$. In this study, we found that the $\mathrm{Wnt} / \beta$-catenin signaling pathway ligand Wnt3a was significantly overexpressed in BM-MSCs from the patients with active cGVHD, leading to the decreased of the phosphor$\beta$-catenin and the dysfunction of osteogenesis and adipogenesis. We further demonstrated that the overexpression of Wnt3a was associated with the severity of cGVHD. With the block of Wnt3a by DKK1, the dysfunction of osteogenesis and adipogenesis in active cGVHD MSCs were restored. Based on these results, we demonstrated that upregulation of Wnt3a and subsequently downstream protein abnormal through $\mathrm{Wnt} / \beta$-catinin signaling pathway participates in the dysfunction of MSCs in patients with active cGVHD. A potential mechanism leading to the dysregulation of Wnt/ $\beta$-catinin signaling pathway might be the infiltration of proinflammatory cytokines, because growing evidence showed that the overexpression of Wnt ligand induced by proinflammatory cytokines contributes to the pathogenesis of autoimmune diseases ${ }^{25,42,43}$. In this study, we found the proinflammatory cytokines, such as IL-6, IL17 , IFN- $\gamma$, and TNF- $\alpha$, were significantly increased in the bone marrow serum. But the relationship between inflammatory cytokines and $\mathrm{Wnt} / \beta$-catinin signaling pathway in the bone marrow of cGVHD patients remains unclear, and the underlying mechanism leading to the dysregulation of the Wnt/ $\beta$-catinin pathway in cGVHD-MSCs needs further explored. Our findings indicated that the differentiation of BM-MSCs in active cGVHD patients were abnormal through the Wnt/ $\beta$-catinin pathway, these abnormalities may contribute to the development of cGVHD. DKK1, the inhibitor of the Wnt pathway, can reverse these abnormalities, thus presenting a novel therapeutic target for cGVHD. Furthermore, the abnormality of BM-MSCs may become one of the indicators for predicting $\mathrm{CGVHD}$ and evaluating the severity of cGVHD.

\section{Conclusion}

In conclusion, the adipogenic and osteogenic capabilities of BM-MSCs were abnormal in the patients with active cGVHD. The dysfunctions of BM-MSCs were mediated by the upregulated of Wnt3a through Wnt/ $\beta$-catenin signaling pathway.

\section{Material and methods \\ Study design and patient eligibility}

The study included 33 patients who were older than 14 years old and developed primary cGVHD after hematopoietic stem cell transplantation at Nanfang Hospital. Thirty-three patients without cGVHD were closely matched to the cGVHD cohort according to time after transplantation, age, gender, stem cell source, conditioning regimen, GVHD prophylaxis and grade of acute GVHD. An additional 7 healthy stem cell transplant donors were recruited for this study. Fresh bone marrow samples were collected from the outpatient bone marrow aspiration. Diagnosis of cGVHD and its severity were established at the time of sample collection using recent National Institutes of Health guidelines ${ }^{44}$. Patients with active cGVHD were defined as requiring prednisone or multiagent immunosuppression ${ }^{45}$. Patients with no cGVHD were defined as those who never developed cGVHD after HSCT. Exclusion criteria were as follows: (1) Patients with inactive cGVHD, defined as a complete response to immune-suppressive therapy for active cGVHD; (2) Patients with recurrent, persistent, or late onset acute GVHD, defined as features of classic acute GVHD occurring beyond 100 days post-transplantation or DLI. This study was conducted in accordance with the Declaration of Helsinki and approved by the institutional review board of Nanfang Hospital (Ethical approval No. NCT04692376). All patients and healthy donors gave written informed consent to participate in the study.

\section{Transplants}

HLA-haploidentical donor (HID) patients were transplanted with a combination of bone marrow (BM) and peripheral blood stem cell (PBSC) grafts, whereas HLAmatched sibling donor (MSD) patients and HLA-matched unrelated donor (MUD) patients received PBSC grafts ${ }^{46}$. Cyclosporine A (CsA), methotrexate (MTX), and mycophenolate mofetil (MMF) were administered to patients undergoing MSD transplant for GVHD prophylaxis. CsA, MTX, MMF and antithymocyte globulin (ATG) were administered to patients undergoing HID transplants for GVHD prophylaxis, and CsA, MTX, and ATG were administered to patients undergoing MUD transplants ${ }^{46}$. aGVHD and cGVHD treatments were detailed in previous report ${ }^{47}$.

\section{Isolation, culture and identification of MSCs}

Isolation, culture, and identification of human MSCs were as described in our previous publication ${ }^{18}$. Briefly, BM samples were collected from participants, and mononuclear cells (BMMNCs)were isolated by Ficoll gradients (Ficoll-PaqueTBD Science, Tianjin, China). 
The cells were cultured with Dulbecco's modified eagle medium nutrient mixture F-12 (Gibco, San Diego, CA) containing $10 \%$ fetal bovine serum (Gibco, New York, US). When cells reached $80-90 \%$ confluence, they were trypsinized by trypsin-EDTA (Gibco, San Diego, CA) and designated as passage 1 . These cells were further passaged at a ratio of 1:3. Passages 2 and 3 MSCs were used for this study. MSCs were identified by antigen expression with flow cytometry as well as by their adipogenic and osteogenic differentiation capacities ${ }^{18}$.

\section{MSCs frequency in BMMNCs}

The MSCs frequency was defined as the number of colony-forming unit fibroblasts (CFU-F) per $10^{6}$ $\mathrm{BMMNCs}^{48}$. Briefly, the BMMNCs were seeded in 6-well plates at a density of $1 \times 10^{5}$ cells/well, cultured for 7 days, and then the numbers of colonies were calculated.

\section{Flow cytometry analysis}

The antibodies and reagents used for flow cytometry analysis were as follows: PE-CD19 (HIB19), APC-CD29 (TS2/16), PE-CD31 (PECAM-1), PE-CD34 (QBEND/10), PERCP5.5-CD44 (IM7), FITC-CD45 (HI30), PECY7CD90 (Thy-1) and PE-CD105 (SN6) were purchased from ThermoFisher Scientific (San Diego, CA). All staining was performed according to the manufacturer's instructions. Flow cytometric analysis was performed on a FACS Canto $^{\mathrm{TM}}$ II Flow Cytometer (BD Biosciences, San Diego, CA), and the resulting data were analyzed with FlowJo software (Tree Star, Ashland, OR).

\section{MSCs proliferation and apoptosis assay}

MSCs proliferation was measured using the Cell Counting Kit-8 according to the manufacturer's protocol (Yeasen, Shanghai, China). An annexin V-fluorescein isothiocyanate (FITC)/propidium iodide (PI) cell apoptosis kit (Yeasen, Shanghai, China) was used to quantify cell apoptosis. The percentages of early apoptotic cells (annexin $\mathrm{V}+/ \mathrm{PI}-$ ) and late apoptotic cells(annexin $\mathrm{V}+/ \mathrm{PI}+$ ) were analyzed using FACSCanto $^{\mathrm{TM}}$ II flow cytometry (BD Biosciences, San Diego, CA).

\section{MSCs differentiation assays}

For osteogenic and adipogenic differentiation of MSCs in vitro, $60 \%-70 \%$ confluent MSCs were cultured in osteogenic or adipogenic medium (Cyagen Biosciences, Santa Clara, CA)for differentiation. Alizarin red staining was used to evaluate calcium deposition while Oil Red O was used to visualize lipid vacuoles.

\section{RNA isolation and real-time quantitative polymerase chain reaction (RT-qPCR) analysis}

RNA was isolated from cells using RNAiso Plus (Takara Bio Inc., Shiga, Japan) and complementary DNA (cDNA) was prepared using a Hifart II First Strand cDNA Synthesis Kit (Yeasen, Shanghai, China) according to the manufacturer's instructions. The cDNA thus obtained was subjected to real-time quantitative PCR with the Hieff qPCR SYBR Green Master Mix kit (Yeasen, Shanghai, China) using the primers. Each sample was analyzed in triplicate and all results were normalized to the expression of glyceraldehyde 3-phosphate dehydrogenase (GAPDH). The primers used are as follows:

PPAR $\gamma$, forward: 5'-AGCCTGCGAAAGCCTTTTGGT G-3',

reverse: 5'-GGCTTCACATTCAGCAAACCTGG-3'. Runx2, forward: 5'-GCAGTTCCCAAGCATTTCAT-3', reverse: $5^{\prime}$-CTGGCGGGGTGTAAGTAAAG-3'

FABP4, forward: 5'-GCAGAAATGGGATGGAAAAT C-3',

reverse: 5'-CTCATAAACTCTCGTGGAAGTGA-3'. Col1a1, forward: 5'-CTGCTGGACGTCCTGGTGAA-3', reverse: $5^{\prime}$-ACGCTGTCCAGCAATACCTTGAG-3'. GAPDH, forward 5'-AGAAGGCTGGGGCTCATTTG-3', reverse 5'-AGGGGCCATCCACAGTCTTC-3'.

\section{Western blot analysis}

Western blot analyses were conducted on the protein of lysates from in vitro cultured MSCs. In the Dickkopf related protein 1 (DKK1) groups, $100 \mathrm{ng} / \mathrm{mL}$ DKK1 (R\&D Systems, USA) was directly added to the cells and incubated for $72 \mathrm{~h}$. The cells were lysed in RIPA lysis buffer containing proteinase inhibitor and PhosSTOP (Fabio Science, China). Equivalent amounts of cell lysate were separated by SDS-polyacrylamide gel electrophoresis (PAGE) and blotted on polyvinylidene fluoride membrane (Millipore Corp, Oakville, Canada). After blocking in 5\% skim milk, the membranes were incubated with primary antibodies and corresponding secondary antibodies. $\beta$-Tubulin was used as an endogenous control to normalize protein expression level. Specific antibodies were incubated as follows: GSK-3 $\beta$ (D5C5Z), Phospho-GSK-3 $\beta$ (Ser9), $\beta$-catenin (D10A8), phospho- $\beta$-catenin (Thr41), Akt (C67E7), p-Akt (Ser473), p38 MAPK (D13E1), p-p38 MAPK (Thr180/Tyr182), NF-kB p65 (D14E12), p-NF-kB p65 (Ser536) and Wnt3a(C64F2) (Cell Signaling Technology, Danvers, MA), GAPDH (FD0063) and $\beta$-tubulin (FD0064) (Fude Biological Technology, Hangzhou, China); and horseradish peroxidase-linked secondary antibody (Abcam, Cambridge, UK). Detection was carried out with ECL kits (Millipore Corp, Oakville, Canada) according to the manufacturer's instructions. Images were obtained using a FluorChem HD2 System (Tanon, Shanghai, China).

\section{Enzyme-linked immunosorbent assay (ELISA)}

Bone marrow serum, supernatants from cultures of MSCs or cocultures of MSCs/PBMCs were collected and cytokines were measured using commercially available 
enzyme-linked immunosorbent assay ELISA kits: IL-6 (70-EK106), IL-10 (70-EK110), IL17 (70-EK117), TNF- $\alpha$ (70-EK182), IFN- $\gamma$ (70-EK180) and TGF- $\beta$ (70-EK981; MultiSciences, Hangzhou, China) according to the manufacturer's instructions.

\section{Mixed lymphocyte reaction}

To determine whether MSCs regulate the immune response, MSCs in gradient concentrations $(5 \times 104 /$ well; $0.5 \times 104 /$ well) were seeded in a 96-well round-bottom plate and cultured at $37^{\circ} \mathrm{C}$ in a humidified incubator for $4 \mathrm{~h}$ to allow cell adherence. Stimulator cells were PBMCs irradiated with 3,000 rads. The stimulator cells were mixed with responder cells at a ratio of 1:10 in MLR culture medium, and then added to the MSCs pre-seeded plates or the concentrated conditioned medium collected from MSCs. The ratio of MSCs to responder cells was 1:10 and $1: 100$. After $72 \mathrm{~h}$ of coculture, cell proliferation was quantified using a CCK-8 according to the manufacturer's instructions.

\section{Statistical analysis}

A descriptive analysis of all variables was performed, including median, range, minimum and maximum values for continuous variables and numbers and frequencies for categorical variables. For categorical variables, the chisquare statistic or Fisher exact test was used to establish differences in their distribution; the Wilcoxon rank-sum test was used to compare continuous variables. Comparisons of three groups were analyzed using one-way ANOVA test. Comparison of two groups was analyzed using an unpaired two-tailed Student's $t$ test. All data were analyzed with GraphPad Software (Prism Version 6.0; GraphPad Software, San Diego, CA) and SPSS version 22.0 (SPSS, Chicago, USA). Statistical significance was defined as $P$ value $<0.05$.

\section{Acknowledgements}

The authors thank Dr. Zheng-shan Yi and his staff for their technical supports at the clinical laboratory of Department of Hematology, Nanfang Hospital.

\section{Author contributions}

H.-Z.Q. performed research, analyzed the data, and wrote the manuscript; Y.-L.Y. and Y.S. performed experiments, collected and analyzed the data; H.Q. performed experiments and wrote the revised manuscript; H.-Y.Z., K.-B.Y., Z.P.F., F.H., L.X., and Y.-Q.C. assisted in the research; H.J. and Q.F.L. designed study, supervised the research and critically revised the manuscript; all authors approved the final version of publication.

\section{Funding}

This work was supported by the National Key Research and Development Projects (Grant No. 2017YFA0105500; 2017YFA0105501 and 2017YFA0105504), the National Natural Science Foundation of China (Grant Nos. 81970161, 81870144, 81700176, and 81770190), Special Project for Research and Development in Key areas of Guangdong Province (Grant No. 2019B020236004), Guangzhou Science and Technology Plan Project (Zhujiang Science and Technology Star Project, No. 201906010094).

\section{Ethics statement}

Bone marrow collection and detection described in this manuscript were approved by the local Ethics committee (Ethical approval No. NCT04692376) and conducted in accordance with the Helsinki declaration. All patients and healthy donors gave written informed consent to participate in the study.

\section{Conflict of interest}

None of the authors has any potential financial conflict of interest related to this manuscript. The material in our manuscript is original research, has not been previously published and has not been submitted for publication elsewhere while under consideration.

\section{Publisher's note}

Springer Nature remains neutral with regard to jurisdictional claims in published maps and institutional affiliations.

Supplementary information The online version contains supplementary material available at https://doi.org/10.1038/s41419-021-03570-6.

Received: 20 November 2020 Revised: 25 February 2021 Accepted: 27 February 2021

Published online: 23 March 2021

\section{References}

1. Horwitz, M. E. \& Sullivan, K. M. Chronic graft-versus-host disease. Blood Rev. 20, 15-27 (2006).

2. Bolaños-Meade, J. et al. Three prophylaxis regimens (tacrolimus, mycophenolate mofetil, and cyclophosphamide; tacrolimus, methotrexate, and bortezomib; or tacrolimus, methotrexate, and maraviroc) versus tacrolimus and methotrexate for prevention of graft-versus-host disease with haemopoietic cell transplantation with reduced-intensity conditioning: a randomised phase 2 trial with a non-randomised contemporaneous control group (BMT CTN 1203). Lancet Haematol. 6, e132-e143 (2019).

3. Shouval, R. et al. Outcomes of allogeneic haematopoietic stem cell transplantation from HLA-matched and alternative donors: a European Society for Blood and Marrow Transplantation registry retrospective analysis. Lancet Haematol. 6, e573-e584 (2019).

4. Bazarbachi, A. H. et al. Allogeneic stem-cell transplantation with sequential conditioning in adult patients with refractory or relapsed acute lymphoblastic leukemia: a report from the EBMT Acute Leukemia Working Party. Bone marrow Transplant. 55, 595-602 (2020).

5. Jin, H. et al. Invasive fungal disease is associated with chronic graft-versus-host disease after allogeneic hematopoietic stem cell transplant: a single center, retrospective study. Infection 47, 275-284 (2019).

6. MacDonald, K. P., Hill, G. R. \& Blazar, B. R. Chronic graft-versus-host disease: biological insights from preclinical and clinical studies. Blood 129, 13-21 (2017).

7. Blazar, B. R., Murphy, W. J. \& Abedi, M. Advances in graft-versus-host disease biology and therapy. Nat. Rev. Immunol. 12, 443-458 (2012).

8. Zeiser, R. \& Blazar, B. R. Pathophysiology of chronic graft-versus-host disease and therapeutic targets. N. Engl. J. Med. 377, 2565-2579 (2017).

9. Jin, H. et al. Antibodies from donor B cells perpetuate cutaneous chronic graftversus-host disease in mice. Blood 127, 2249-2260 (2016).

10. Jin, H. et al. Expansion of circulating extrafollicular helper T-like cells in patients with chronic graft-versus-host disease. J. Autoimmun. 100, 95-104 (2019).

11. Shono, Y. et al. Bone marrow graft-versus-host disease: evaluation of its clinical impact on disrupted hematopoiesis after allogeneic hematopoietic stem cell transplantation. Biol. Blood Marrow Transplant. 20, 495-500 (2014).

12. Biedermann, B. C. et al. Endothelial injury mediated by cytotoxic T lymphocytes and loss of microvessels in chronic graft versus host disease. Lancet $\mathbf{3 5 9}$, 2078-2083 (2002)

13. Mosna, F., Sensebé, L. \& Krampera, M. Human bone marrow and adipose tissue mesenchymal stem cells: a user's guide. Stem Cells Dev. 19, 1449-1470 (2010).

14. Meierhenry, J. A. et al. Placenta as a source of stem cells for regenerative medicine. Curr. Pathobiol. Rep. 3, 9-16 (2015).

15. Feng, Y. et al. Mesenchymal stromal cells-derived matrix Gla protein contribute to the alleviation of experimental colitis. Cell Death Dis. 9, 691 (2018). 
16. Mimeault, M., Hauke, R. \& Batra, S. K. Stem cells: a revolution in therapeuticsrecent advances in stem cell biology and their therapeutic applications in regenerative medicine and cancer therapies. Clin. Pharmacol. Therapeutics $\mathbf{8 2}$, 252-264 (2007)

17. Charbord, P. Bone marrow mesenchymal stem cells: historical overview and concepts. Hum. Gene Ther. 21, 1045-1056 (2010).

18. Zhao, K. et al. Immunomodulation effects of mesenchymal stromal cells on acute graft-versus-host disease after hematopoietic stem cell transplantation. Biol. Blood. Marrow Transplant. 21, 97-104 (2015).

19. Ji, J. et al. JAK-STAT signaling mediates the senescence of bone marrowmesenchymal stem cells from systemic lupus erythematosus patients. Acta Biochim. Biophys. Sin. (Shanghai) 49, 208-215 (2017).

20. Zhen, $G$. et al. Inhibition of TGF- $\beta$ signaling in mesenchymal stem cells of subchondral bone attenuates osteoarthritis. Nat. Med. 19, 704-712 (2013).

21. Xie, Z. et al. Imbalance between bone morphogenetic protein 2 and noggin induces abnormal osteogenic differentiation of mesenchymal stem cells in ankylosing spondylitis. Arthritis Rheumatol. 68, 430-440 (2016).

22. Medinger, $\mathbf{M}$. et al. Numerical impairment of nestin(+) bone marrow niches in acute GvHD after allogeneic hematopoietic stem cell transplantation for AML. Bone Marrow Transplant. 50, 1453-1458 (2015).

23. Ding, L. et al. Tumor necrosis factor $a$ in $\mathrm{aGVHD}$ patients contributed to the impairment of recipient bone marrow MSC stemness and deficiency of their hematopoiesis-promotion capacity. Stem Cell Res. Ther. 11, 119 (2020).

24. Ishikawa, Y. Wnt signaling and orthopedic diseases. Am. J. Pathol. 167, 1-3 (2005).

25. Briolay, A. et al. Autocrine stimulation of osteoblast activity by Wnt5a in response to TNF-a in human mesenchymal stem cells. Biochem. Biophys. Res. Commun. 430, 1072-1077 (2013).

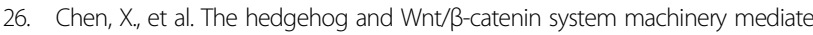
myofibroblast differentiation of LR-MSCs in pulmonary fibrogenesis. Cell Death Dis. 9, 639 (2018).

27. Que, J., Lian, Q., El Oakley, R. M., Lim, B. \& Lim, S. K. PI3 KNAkt/mTOR-mediated translational control regulates proliferation and differentiation of lineagerestricted RoSH stem cell lines. J. Mol. Signal. 2, 9 (2007).

28. James, A. W. et al. Sonic Hedgehog influences the balance of osteogenesis and adipogenesis in mouse adipose-derived stromal cells. Tissue Eng. Part A 16, 2605-2616 (2010).

29. Xu, C., Liu, H., He, Y., Li, Y. \& He, X. Endothelial progenitor cells promote osteogenic differentiation in co-cultured with mesenchymal stem cells via the MAPK-dependent pathway. Stem Cell Res. Ther. 11, 537 (2020).

30. Ding, L. et al. Mesenchymal stem cells in grafts failed to engraft in the bone marrow microenvironment of a leukemia patient post HLA-match and haploidentical allogeneic hematopoietic stem cell transplantations. Pediatr. Hematol. Oncol. 31, 389-391 (2014)

31. Wang, B. et al. Phenotypical and functional characterization of bone marrow mesenchymal stem cells in patients with chronic graft-versus-host disease. Biol. Blood Marrow Transplant. 21, 1020-1028 (2015).
32. Gao, F. et al. Mesenchymal stem cells and immunomodulation: current status and future prospects. Cell Death Dis. 7, e2062 (2016).

33. Ding, $Y$. et al. Rap1 deficiency-provoked paracrine dysfunction impairs immunosuppressive potency of mesenchymal stem cells in allograft rejection of heart transplantation. Cell Death Dis. 9, 386 (2018).

34. Mutt, S. J. et al. Inhibition of cytokine secretion from adipocytes by 1,25dihydroxyvitamin $D_{3}$ via the NF-KB pathway. FASEB J. 26, 4400-4407 (2012).

35. Kim, W., Kim, M. \& Jho, E. H. Wnt/ $\beta$-catenin signalling: from plasma membrane to nucleus. Biochem. J. 450, 9-21 (2013).

36. Niehrs, C. The complex world of WNT receptor signalling. Nat. Rev. Mol. cell Biol. 13, 767-779 (2012).

37. Galli, C. et al. GSK3b-inhibitor lithium chloride enhances activation of Wnt canonical signaling and osteoblast differentiation on hydrophilic titanium surfaces. Clin. Oral. Implants Res. 24, 921-927 (2013).

38. Li, H-X, Luo, X, Liu, R-X, Yang, Y-J, \& Yang, G.-S. Roles of Wnt/beta-catenin signaling in adipogenic differentiation potential of adipose-derived mesenchymal stem cells. Mol. Cell. Endocrinol. 291, 116-124 (2008).

39. Nakamura, Y., Nawata, M. \& Wakitani, S. Expression profiles and functional analyses of Wnt-related genes in human joint disorders. Am J Pathol 167, 97-105 (2005).

40. Wang, X.-D., Huang, X.-F. \& Yan, Q.-R. Bao C-d. Aberrant activation of the WNT/ $\beta$-catenin signaling pathway in lupus nephritis. PLOS ONE 9, e84852 (2014).

41. Chen, C.-W. et al. Pharmacological inhibition of porcupine induces regression of experimental skin fibrosis by targeting Wnt signalling. Ann. Rheum. Dis. 76, 773-778 (2017).

42. Assassi, S. \& Mayes, M. D. What does global gene expression profiling tell us about the pathogenesis of systemic sclerosis? Curr. Opin. Rheumatol 25, 686-691 (2013).

43. von Toerne, C. et al. Effector T cells driving monophasic vs. relapsing/remitting experimental autoimmune uveitis show unique pathway signatures. Mol Immunol. 48, 272-280 (2010).

44. Jagasia, M. H. et al. National Institutes of Health Consensus Development Project on Criteria for Clinical Trials in Chronic Graft-versus-Host Disease: I. 2014 Diagnosis Staging Working Group report. Biol. Blood Marrow Transplant. 21 389-401.e1 (2015).

45. Allen, J. L. et al. Increased BCR responsiveness in B cells from patients with chronic GVHD. Blood 123, 2108-2115 (2014).

46. Xuan, L. et al. Sorafenib maintenance in patients with FLT3-ITD acute myeloid leukaemia undergoing allogeneic haematopoietic stem-cell transplantation: an open-label, multicentre, randomised phase 3 trial. Lancet Oncol. 21 1201-1212 (2020).

47. Lin, R. et al. Two dose levels of rabbit antithymocyte globulin as graft-versushost disease prophylaxis in haploidentical stem cell transplantation: a multicenter randomized study. BMC Med. 17, 156 (2019).

48. Larghero, J. et al. Phenotypical and functional characteristics of in vitro expanded bone marrow mesenchymal stem cells from patients with systemic sclerosis. Ann. Rheum. Dis. 67, 443-449 (2008). 